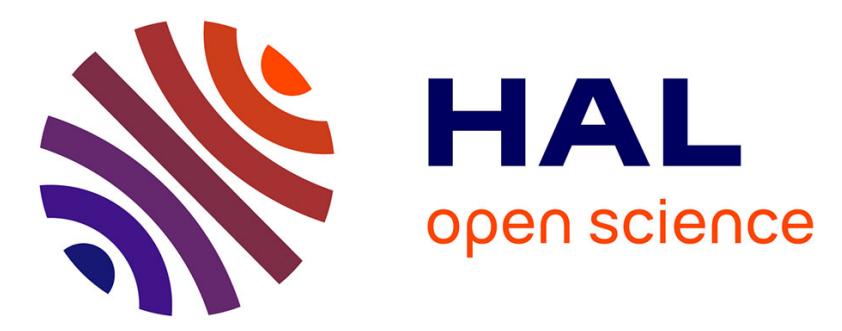

\title{
Data-driven prognostic method based on Bayesian approaches for direct remaining useful life prediction.
}

Ahmed Mosallam, Kamal Medjaher, Noureddine Zerhouni

\section{To cite this version:}

Ahmed Mosallam, Kamal Medjaher, Noureddine Zerhouni. Data-driven prognostic method based on Bayesian approaches for direct remaining useful life prediction.. Journal of Intelligent Manufacturing, 2014, pp.1-20. 10.1007/s10845-014-0933-4 . hal-01025442

\section{HAL Id: hal-01025442 \\ https://hal.science/hal-01025442}

Submitted on 17 Jul 2014

HAL is a multi-disciplinary open access archive for the deposit and dissemination of scientific research documents, whether they are published or not. The documents may come from teaching and research institutions in France or abroad, or from public or private research centers.
L'archive ouverte pluridisciplinaire $\mathbf{H A L}$, est destinée au dépôt et à la diffusion de documents scientifiques de niveau recherche, publiés ou non, émanant des établissements d'enseignement et de recherche français ou étrangers, des laboratoires publics ou privés. 


\title{
Data-driven prognostic method based on Bayesian approaches for direct remaining useful life prediction
}

\author{
A. Mosallam - K. Medjaher · N. \\ Zerhouni
}

Received: date / Accepted: date

\begin{abstract}
Reliability of prognostics and health management systems (PHM) relies upon accurate understanding of critical components' degradation process to predict the remaining useful life (RUL). Traditionally, degradation process is represented in the form of data or expert models. Such models require extensive experimentation and verification that are not always feasible. Another approach that builds up knowledge about the system degradation over the time from component sensor data is known as data driven. Data driven models, however, require that sufficient historical data have been collected. In this paper, a two phases data driven method for RUL prediction is presented. In the offline phase, the proposed method builds on finding variables that contain information about the degradation behavior using unsupervised variable selection method. Different health indicators (HI) are constructed from the selected variables, which represent the degradation as a function of time, and saved in the offline database as reference models. In the online phase, the method finds the most similar offline health indicator, to the online health indicator, using k-nearest neighbors (k-NN) classifier to use it as a RUL predictor. The method finally estimates the degradation state using discrete Bayesian
\end{abstract}

\author{
Ahmed Mosallam \\ FEMTO-ST Institute, AS2M department, \\ University of Franche-Comté/CNRS/ENSMM/UTBM, \\ 24 rue Alain Savary, 25000 Besançon, France. \\ E-mail: ahmed.mosallam@femto-st.fr \\ Kamal Medjaher \\ FEMTO-ST Institute, AS2M department, \\ University of Franche-Comté/CNRS/ENSMM/UTBM, \\ 24 rue Alain Savary, 25000 Besançon, France. \\ E-mail: kamal.medjaher@ens2m.fr \\ Noureddine Zerhouni \\ FEMTO-ST Institute, AS2M department, \\ University of Franche-Comté/CNRS/ENSMM/UTBM, \\ 24 rue Alain Savary, 25000 Besançon, France. \\ E-mail: noureddine.zerhouni@ens2m.fr
}


filter. The method is verified using battery and turbofan engine degradation simulation data acquired from NASA data repository. The results show the effectiveness of the method in predicting the RUL for both applications.

Keywords Degradation Modeling · Online Estimation · Discrete Bayes Filter · Uncertainty Representation · Data-driven PHM.

\section{Introduction}

The large volume of data gathered continuously from different systems has created challenges to interpret such data in order to anticipate the breakdowns. Most large industries have specialized engineers whom are skilled in the use of high technology maintenance equipment and have earned special certification in the field of maintenance. Nevertheless, it is still hard to take immediate decisions and predict the system failure. The need of computer systems that constantly record and analyze data to predict the RUL of critical components is particularly important for facilitating maintenance decisions.

In general, maintenance involves performing routine actions to obtain optimal availability of industrial systems [1]. Maintenance routines can be broadly categorized into two main types, namely, corrective and preventive maintenance [2]. In corrective maintenance, the interventions are performed only when the critical component is fully worn out and failure occurred. Preventive maintenance can be further divided into two main approaches, namely, time-based maintenance and condition based maintenance (CBM). In timebased maintenance, the interventions are placed according to periodic intervals regardless of the assets' health condition and thus the service life of the critical components is not fully utilized [3]. Condition based maintenance uses machine run-time data to assess the critical component's state and schedule required maintenance actions prior to breakdown [4]. Furthermore, predictive maintenance utilizes the current health status of a given critical component to predict its future condition and plan maintenance actions. Prognostics and Health Management (PHM) [5] is a process which links degradation modeling research to predictive maintenance policies.

Prognostics and health management consists of four main modules: fault detection, fault diagnostics, fault prognostics and decision making [6]. Fault detection can be defined as the process of recognizing that a problem has occurred regardless of the root cause [7]. Fault diagnostics is the process of identifying the faults and their causes [8]. Fault prognostics can be defined as the prediction of when a failure might take place [9]. Finally, decision making step uses all the information gathered about the monitored system status to choose the optimal maintenance actions [10]. Among other routines, prognostics have recently attracted significant research interest due to the need of models for accurate RUL prediction for different applications.

RUL prediction of critical components is a non-trivial task for many reasons. Sensor signals, for instance, are usually obscured by noise and thus it is very challenging to process and to extract informative representation of the 
RUL [11]. Another problem is the prediction uncertainty due to the variation of the end of life time that can differ for two components made by the same manufacturer and operating under the same conditions. Therefore, proposed models should include such uncertainties and represent them in a probabilistic form $[12,13]$.

RUL prediction models can be realized using two different methods, namely, physics based and data-driven methods [14]. Physics based methods build physical models of the desired critical components by the means of state-space models [15] and dynamic ordinary or partial differential equations [16]. These models require extensive experimentation and model verification [17]. However, these models are very reliable at least until the system is upgraded or changed [18]. Data-driven methods can be used when the first principles of the system operation are complex such that developing an accurate physics of failure model is not feasible $[19,20]$. Such methods employ pattern recognition and machine learning techniques to characterize the desired critical components' degradation behavior [21]. One way to do data-driven RUL prediction is by first estimating the current health status of the desired component and when the degradation exceeds the alarm threshold, the algorithms start predicting the RUL [39-41]. Different regression models have been proposed in the literature to deal with data-driven RUL prediction problem such as the auto regressive model and the multivariate adaptive regression splines [22-27]. A drawback of using regression methods is that when available component degradation history is incomplete the extrapolation may lead to large errors [42]. There have been more interests lately on various types of neural networks and neural-fuzzy systems [28-38]. However, these methods generate black box models and it is difficult to select the structure of the network [43]. Similaritybased methods are shown to be very effective in performing RUL prediction. A similarity-based method based on linear regression to construct offline degradation models is proposed in [42]. The method measures the similarity between test instance and offline models and the selected offline instance is used for RUL prediction. The RUL probability density of the test instance is estimated from the multiple local predictions using the kernel density estimation method. The main problem with this method is the manual selection of the informative sensor data. Another similarity-based method that utilizes k-NN and belief function theory to estimate the health and from that deduce the RUL of turbofan engines is proposed in [43]. The authors manually annotate the health status of the offline data sets and then the method predicts the RUL when the degradation level reaches a predefined alarm threshold.

Alternatively, instead of learning the degradation from the data and predict the RUL; direct RUL prediction algorithms learn the relation between the sensor data and the end of life to predict the RUL. To do this, health indicators are extracted from the raw monitoring signals, which may have originated from single sensor or from a number of sensors aggregated to represent the degradation evolution over time. Although this type of RUL prediction is relatively easy to implement, there are few published examples in the literature [44]. 
In this work, direct RUL prediction method is presented. The aim of this work is to model the relation between sensor data and end of life to predict the RUL without the need for predefined alarm threshold. The method builds on extracting health indicators from the training data, which are used as reference models. For new data, the method finds in the database the most similar signal to be used as a RUL predictor. The method then estimates the new signal's health status using a Bayesian approach.

The assumptions taken in this work can be summarized as follows:

1. The method can only be applied to critical components, which are already identified by the system expert.

2. Historical data should contain degradation evolution of the critical component over time.

3. Historical data should contain sufficient number of training instances to build representative models of the desired critical component's behavior.

4. The predicted RUL values will span between the values available in the offline data sets.

This paper is structured as follows. Section 2 presents the proposed method. The experimental set-up and the simulation results are depicted in Section 3. Finally, Section 4 concludes the paper.

\section{Data-driven prognostic method based on Bayesian approaches for direct remaining useful life prediction}

Measurements observed from monitored components are usually noisy multidimensional time series signals. Thus, it is essential in the offline phase to first extract information that represents the degradation evolution over time. The relation between the extracted information and the end of life should be modeled to predict the RUL. To do this, the proposed method selects interesting sensor signals and builds health indicators that are used as offline models. In the online phase, the method estimates the current status from the unseen online data, using only the sensors selected on the offline phase, and predicts the RUL by measuring the similarity to the offline data. The method is summarized in Figure 1 and explained hereafter.

\subsection{Offline phase}

In order to build offline reference models, representative features should be extracted from the training data. Those features are later labeled with the end of life time and saved in the database. To do that, a trend construction method is applied [45]. The method builds on two main steps, namely, variable selection and health indicator construction. 


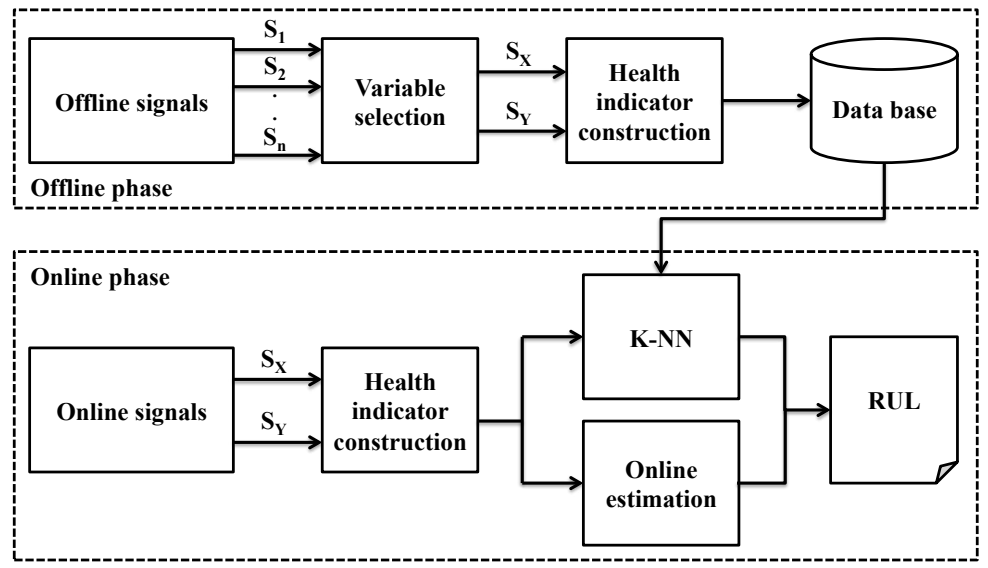

Fig. 1: The method's general scheme.

\subsubsection{Variable selection}

Not all signals from the monitored component are informative. Signals that have non-random relationships contain information about the system degradation. To select such signals, an unsupervised variable selection algorithm based on information theory is applied [46]. The algorithm first calculates pairwise symmetrical uncertainty $(S U)$, as depicted in Figure 2a, for all the input signals:

$$
S U(X, Y)=2 \times \frac{I(X, Y)}{H(X)+H(Y)}
$$

where, $I(X, Y)$ is the mutual information between two random variables $X$ and $Y ; H(X)$ and $H(Y)$ are information entropy values of the random variables $X$ and $Y$, respectively. Then, the algorithm groups the variables based on the SU distance using hierarchical clustering shown in Figure 2b. The algorithm finally ranks the resulting clusters according to the quality of the included signals in representing interesting relationships using normalized self-organizing map distortion measure. A cluster gets low rank if it contains random signals. On the other hand, a cluster gets high rank if it contains signals that exhibit nonrandom relationship and those signals will be used for later processing.

\subsubsection{Health indicator construction}

The following task, after selecting the interesting variables from the initial monitoring raw signals, is to extract smooth monotonic signals, which are correlated with the component's end of life. These monotonic signals are later processed to extract representative features over the time, which can be used 


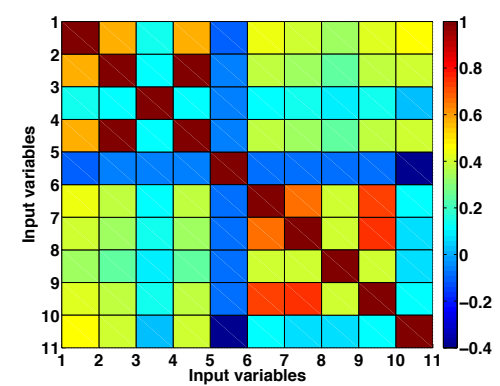

(a) SU similarity matrix.

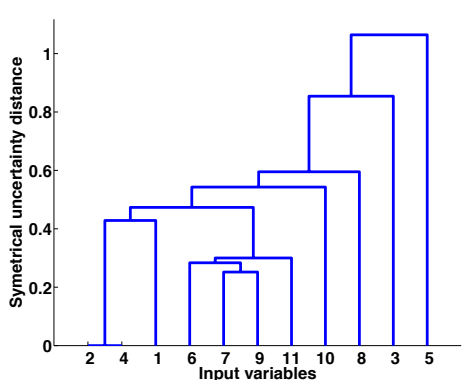

(b) Tree representation of variables relations.

Fig. 2: Variable selection step for 11 sensor signals from NASA battery B0005.

as health indicators and are saved in the database as reference models. To do this, three processing steps, namely variable compression, trend extraction and feature extraction, are applied to the selected variables.

Variable compression: The goal of this step is to compress the $n$ signals selected in the previous step onto one-dimensional space. From each cycle, the selected variables are compressed using standard principal component analysis (PCA) method. The first principle component retains the maximum variance while reducing the dimensionality to one dimension. Therefore, only the first principle component is used to represent the health status evolution with respect to time.

Trend extraction: The compressed variables are then further processed at each cycle to get monotonic trends that can represent the variation of end of life using empirical mode decomposition algorithm (EMD) [47]. EMD is a method employed to decompose a signal into successive intrinsic mode functions (IMF) and a residual signal $r_{n}(t)$, which should be a constant or monotonic signal that can be represented as:

$$
r_{n}(t)=X(t)-\sum_{i=1}^{n} i m f_{i}(t)
$$

where, $X(t)$ is the input signal, $i m f_{i}$ is the IMF and $n$ is the maximum number of IMFs. The generated residual can represent the relation between the generated trends and end of life time. For example, Figure 3a shows an acceleration signal acquired from a degraded bearing that was worn out after around 9 hours and Figure 3b shows a non-degraded bearing were the experiment stopped at the same time of the degraded bearing [48]. EMD was applied to both of the two signals and the resulting residuals are shown in Figure 3c. The experiments show that the residual of the degraded component was a monotonic signal while the non-degraded component generated almost a constant residual. 


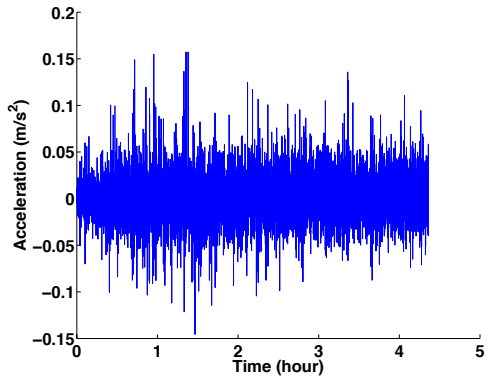

(a) Degraded bearing.

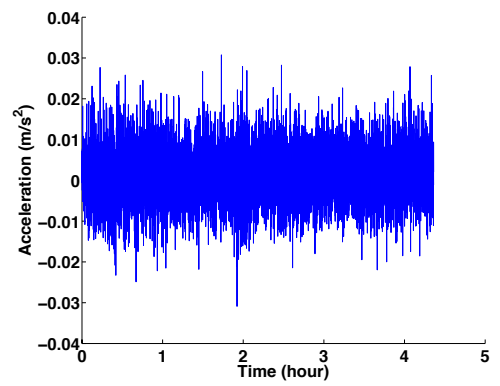

(b) Non-degraded bearing.

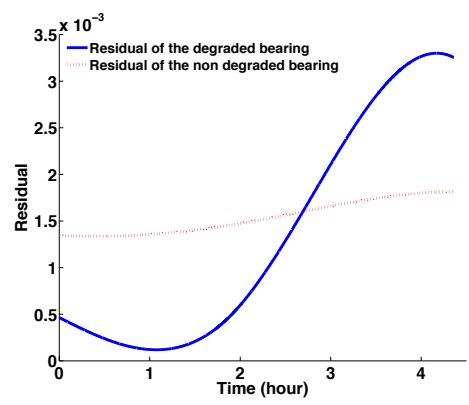

(c) Residual of both bearings.

Fig. 3: Residual variation according to the health status.

Feature extraction: So far, trends are extracted from the compressed variables. These trends should be used to build an offline model, which can be used to classify new online data. In order to make the classification task more efficient, discriminant features should be extracted from acquired trends. Different approaches have been proposed for extracting features such as mean, variance, multi-exponential function, curve fitting, discrete wavelet transform and discrete Fourier transform [49]. However, selecting appropriate features is mainly problem specific. Recalling Figure 3c, the slope of the trend can be a discriminant characteristic of the trend. A trend with more RUL tends to have smaller slope and vice versa. The y-intercept of the curve fit shows the beginning value of the extracted trend, which also can be a discriminant feature. Another discriminant feature for this problem is the mean of the extracted trend. Every data value in the trend contributes to the mean value, and the change of the data over time will affect the mean value. Finally, the variance of the extracted trend describes the spread of a trend with respect to end of life time, which is also an important feature to extract.

In this work, a feature vector $F=\left[a, b, \bar{x}, s^{2}\right]$ is extracted from each trend at each time, where, $a$ and $b$ are the slope and the y-intercept of a linear curve fit of the input trend respectively, $\bar{x}$ and $s^{2}$ are the mean and the variance of the input trend, respectively. The feature vector is extracted from each 
trend starting from time 0 until current time $t$. Figure 4 shows an example of the feature extraction process from three different trends extracted at three constitutive cycles, namely, cycle 40, cycle 100 and cycle 167 . The method extracts the feature vector, from each trend built from in previous step, labels the vector with the cycle number and end of life value and saves it in the offline database. This process is repeated recursively, until the method reaches

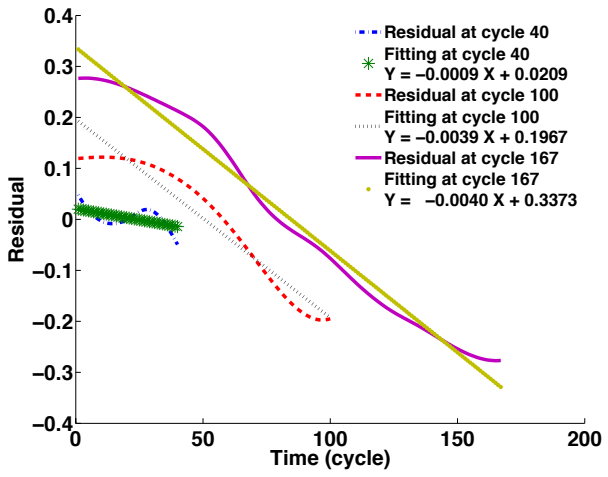

(a) Slope and y-intercept values.

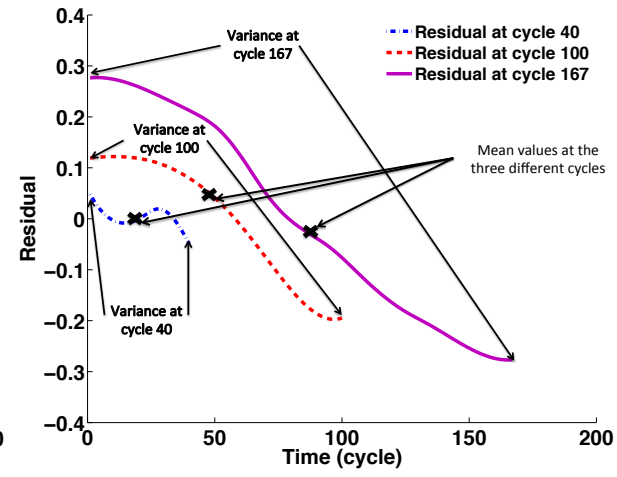

(b) Mean and variance values.

Fig. 4: Feature extraction from input residual at cycles 40, 100 and 167.

the end of life, to generate a representation of the degradation as a function of time. The resulting function or health indicator, as depicted in Figure 5, is then used to represent the corresponding critical component according to its end of life time. Each group of health indicators with similar end of life time is considered as a class and saved in the offline database.

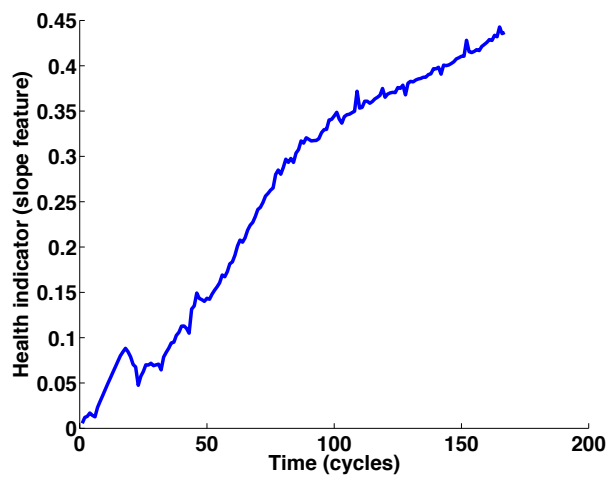

(a) Slope features over time.

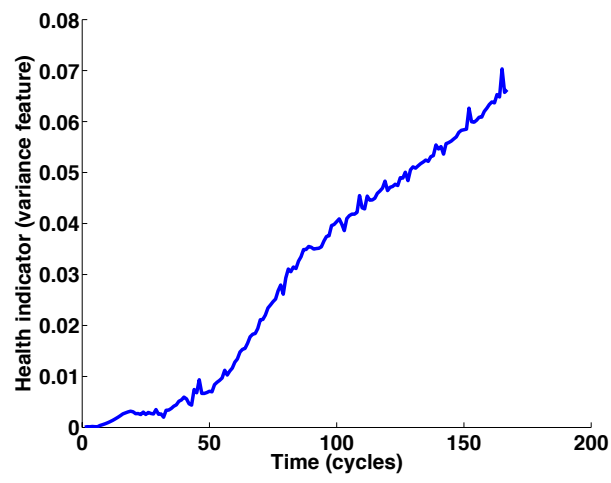

(b) Variance features over time.

Fig. 5: Health indicators constructed from the NASA battery B0005. 


\subsection{Online phase}

In this phase, new sensor data are collected online from the critical component(s) from only the sensors that are selected in the offline phase. The processes applied in the offline phase, such as extracting monotonic trends and feature vector $F$, are applied to the online signals. The generated vector $F$ is then fed to a k-NN classifier to find the most similar offline signal (or case). The end of life value of the offline signal is then considered to be the RUL of the test signal. The online estimator recursively estimates the trends value until it stops at end of life time.

\subsubsection{Classification using $k$-Nearest Neighbours}

In order to build the predictive model, a k-NN classifier is applied in this work. The extracted feature vector $F$ at time $t$ is passed to the $\mathrm{k}-\mathrm{NN}$ to find the most similar offline group in the database at the same time as shown in Figure 6a. The classification decision is based on largest posterior probability of the tested sample at time $t$, therefore, a probability value will be assigned to the prediction output:

$$
p\left(E O L_{k} \mid F_{t}\right)=\frac{p\left(F_{t} \mid E O L_{k}\right) \times p\left(E O L_{k}\right)}{p\left(F_{t}\right)}
$$

where, $F_{t}$ is the online feature vector, $E O L_{k}$ is the class or end of life value for group $k, p\left(F_{t} \mid E O L_{k}\right)$ is the probability of observing $F_{t}$ given $E O L_{k}$, also known as the likelihood, $p\left(E O L_{k}\right)$ is class prior and $p\left(F_{t}\right)$ is the marginal likelihood. The end of life with the highest posterior probability will be used as the end of life for the new signal as depicted in Figure $6 \mathrm{~b}$.

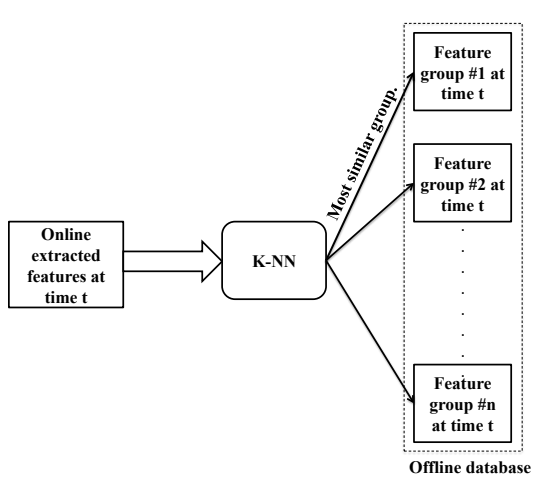

(a) Selecting the most similar group.



(b) End of life for the online signal.

Fig. 6: Finding the end of life for the online signal at time $=50$ cycles using k-NN classifier. 


\subsubsection{Online estimation}

To estimate the actual value of the online health indicator at the predicted end of life value, a recursive discrete Bayesian filter is applied to the online trends. This filter, decomposes the state space into many regions and represents the cumulative posterior for each region by probability values, see Algorithm 1.

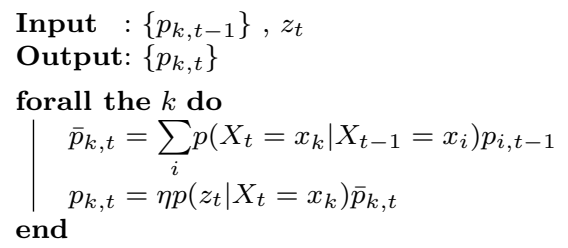

Algorithm 1: Discrete Bayesian filter.

The input to the algorithm is a discrete probability distribution $\left\{p_{k, t}\right\}$ along with the recent measurement $z_{t}$. The first line of the Algorithm 1, $\bar{p}_{k, t}=\sum_{i} p\left(X_{t}=x_{k} \mid X_{t-1}=x_{i}\right) p_{i, t-1}$, calculates the prediction for the new state based on previous state uncertainty and state transition model. The prediction is then updated in the second line, $p_{k, t}=\eta p\left(z_{t} \mid X_{t}=x_{k}\right) \bar{p}_{k, t}$, so as to incorporate the measurement. Discrete Bayesian filters apply to problems with finite state space, where the random variable $X_{t}=x_{1, t} \cup x_{2, t} \cup \ldots x_{k, t}$. A straightforward decomposition of $X_{t}$ is a multidimensional grid, where each $x_{k, t}$ is a bin or region. The size of each bin is $d x=\frac{x_{\max }-x_{\min }}{n}$, where $x_{\max }$ is the maximum state value, $x_{\min }$ is the minimum state value and $n$ is the number of bins. Each bin can then be represented as a Gaussian function with a mean value at each state and a common variance:

$$
p\left(X_{t} \mid X_{t-1}\right)=\left\|d x \times \mathcal{N}\left(X_{k, t}, \sigma^{2}\right)\right\|
$$

where, $p\left(X_{t}=x_{k} \mid X_{t-1}\right)$ is the state transition model, $d x$ is the bin size and $\mathcal{N}\left(X_{k, t}, \sigma^{2}\right)$ is the normal distribution at state $X_{k, t}$. Moreover, Eq. (4) is normalized to turn this quantity into a probability distribution. Similarly, the measurement probability model can be calculated in the same manner as the transition model. Figure 7 shows the final result of the proposed method. The estimation algorithm stops once it reaches the predicted end of life. The uncertainty about the prediction and current status are represented in probabilistic forms. The overall method is summarized in Algorithm 2.

The machine degradation information, i.e. predicted RUL, the estimated health status and corresponding uncertainties, produced by this method can be used as an input for maintenance decision making routine. Decision-making routine considers both machine degradation information and system structure to assist the plant manager in making a dynamic maintenance plan based not only on the optimization of single component/subsystem plan, but also on the global scheduling of whole system for optimized maintenance prioritization 


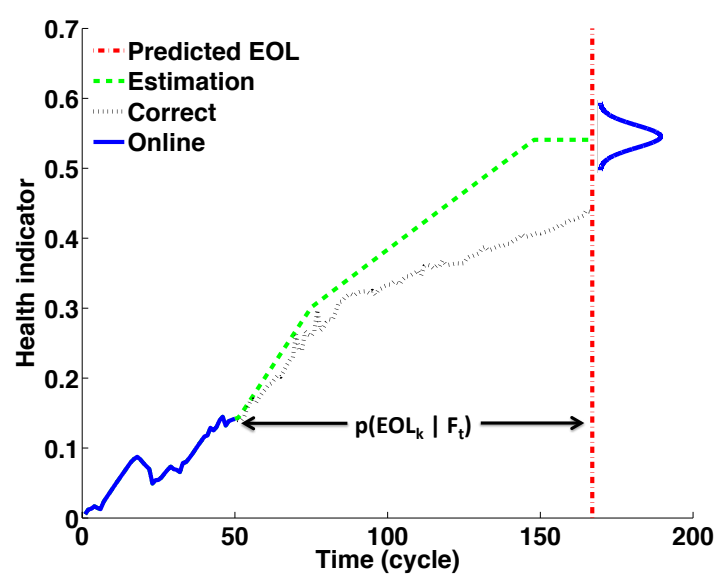

Fig. 7: Estimation of the health indicator using Bayesian filter.

Data: $\{$ trainingData, testData $\}$

Result: $\left\{D_{l}, R U L\right\}$

1 for $\forall$ training Data do

$2 \mid$ selectedVariables $=$ FindBestGroup $($ trainingData $)$;

3 end

4 Offline phase

5 for $i=1$ : numberOf(trainingData) do

$E O L=$ lengthOf $($ trainingData $(i))$;

for $j=2: E O L$ do

selectedVariables $=$ getSelectedVariables $($ trainingData $(i))$;

$i p=$ selectedVariables $(1: j)$

firstComponent $=$ GetFirstComponent $(i p)$;

residual = GetEMDResidual(firstComponent $)$;

features $=$ GetFeatures(residual);

end

$H I=$ append $([$ features,$i])$;

end

$D_{l}=\operatorname{append}([H I, E O L])$

16 end

17 Online phase

18 selectedVariables $=$ getSelectedVariables (testData);

19 firstComponent $=$ GetFirstComponent (selectedVariables);

20 residual $=$ GetEMDResidual(firstComponent);

21 testingFeatures = GetFeatures(residual);

$22 E O L=k N N\left(\right.$ testFeatures, $\left.D_{l}\right)$;

23 rulEstimation $=$ discreteBayesianFilter $($ testFeatures, EOL);

$24 R U L=($ EOL, rulEstimation $)$;

Algorithm 2: The general algorithm of the proposed method

[50]. Maintenance prioritization is crucial and important to reduce unnecessary maintenance activities, especially when availability of maintenance resources are limited [51]. 


\section{Applications and results}

Two real life data sets are used in this work to verify the proposed method: turbofan engine and lithium-ion battery aging data sets.

\subsection{Turbofan engine data}

The turbofan engine data sets are generated using commercial modular aeropropulsion system simulation (C-MAPSS) [53]. They consist of four training files, four testing files and four RUL values files. The training files contain run to failure sensor records of a fleet of engines generated under different combinations of operational conditions and fault modes. Each engine is operating normally and it develops a fault at some point during the operation until finally it reaches the system failure and the engine stops. The test files are generated in the same way; however, the sensor readings are omitted prior to system failure. The RUL files contain vector of true RUL values for the test data. Each training and test file contains 26 columns that represent different variables. The first two columns represent the engine number and the time in cycles, respectively. The next three columns represent the operational settings. The last 21 columns, or variables, represent different time series sensor data such as total temperature at fan inlet, pressure at fan inlet, physical fan speed, etc. Each row represents a data snapshot taken during a single cycle. In this work, the data file "train_FD001.txt" is used for offline training and "test_FD001.txt" is used for online testing. Each file contains data for 100 engines and the objective is to predict the number of remaining operational cycles before failure in the test set. The true RUL values for the test data are presented in the data file "RUL_FD001.txt".

Variable selection: One of the results of the selection algorithm is the pair of sensors number $\{8,13\}$, i.e. physical fan speed and corrected fan speed, respectively (Figure 8a). The selected group is interesting as the two variables are correlated and both are related to the fan speed. Then, the method starts constructing the monotonic trends iteratively from each pair at each time.

Health indicator: As mentioned before, four features are extracted from each trend at each time and labeled with end of life time to be saved in the offline database. The features represent the relation between the extracted trends and the engine's end of life. Figure $8 \mathrm{~b}$ shows one of the four health indicators for the NASA training engine number 61 . The indicator is monotonic and shows how the relation between the end of life and the extracted trend changes through the time. Each health indicator is then saved in offline database and labeled with the end of life time and will be used for predicting the RUL of new sensor data. 


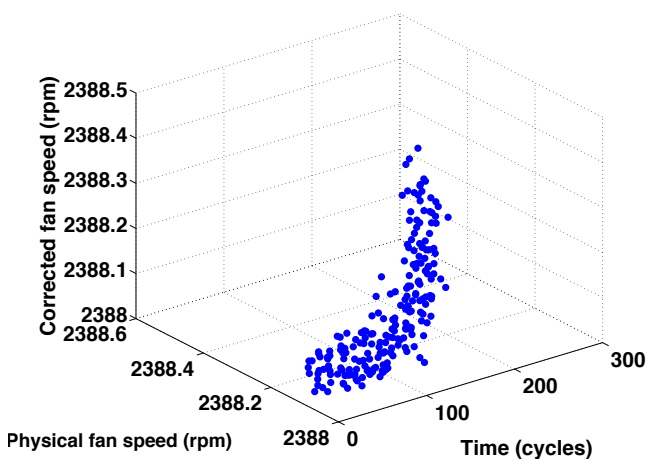

(a) The selected pair of sensors.

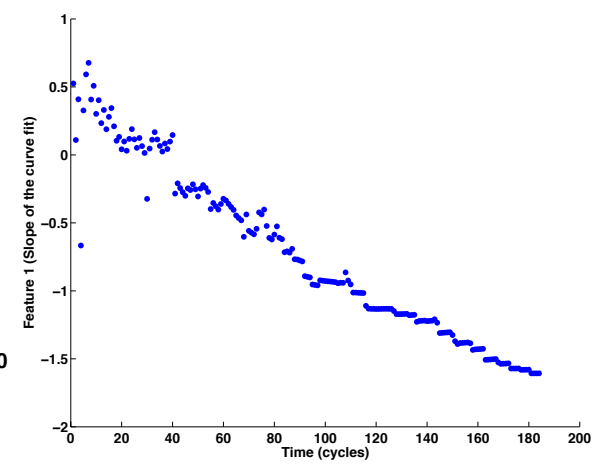

(b) The slope health indicator.

Fig. 8: Results of variable selection and health indicator construction for the NASA turbofan engine 61.

Prediction results: Figure 9 shows the predicted RUL for 4 engines at all cycles. It can be noticed that the accuracy of the predictions increases with the time. The prediction error at the last cycles is less than the errors at the beginning. To assess the performance of the proposed method, the mean absolute percentage error (MAPE) is calculated for all 100 online predictions:

$$
\operatorname{MAPE}(\%)=\frac{100 \%}{n} \times \sum_{i=1}^{n}\left|\frac{R U L_{i}-R U L_{i}^{*}}{R U L_{i}}\right|
$$

where $R U L$ and $R U L^{*}$ are the actual and predicted RUL values respectively and $n$ is the number of total predictions. The error is calculated only for the last cycles of all 100 test signals. The MAPE over the 100 test data equals to $12.19 \%$. And for comparison, the MAPE over the first 15 test engines is $8.7691 \%$, which outperforms the method presented in [34] in which the MAPE value is $15.5 \%$ for the 15 test engines.

\subsection{Lithium-ion battery data}

These data are collected on 34 lithium-ion batteries run through different operational profiles (e.g. charge, discharge and impedance) at different temperatures [52]. In this work only charge and discharge data are used. Each data set, corresponding to one experiment, consists of 11 variables such as charging voltage, charging current, temperature, discharging current, discharging voltage and capacity. The aging of the batteries was accelerated and the experiments continued until the batteries reached their end of life time. Each cycle is presented by the mean value to reduce the processing time. In order to validate the proposed method, a 3-fold cross-validation is applied, i.e. the available data sets are partitioned into three groups of equal size. Each group 


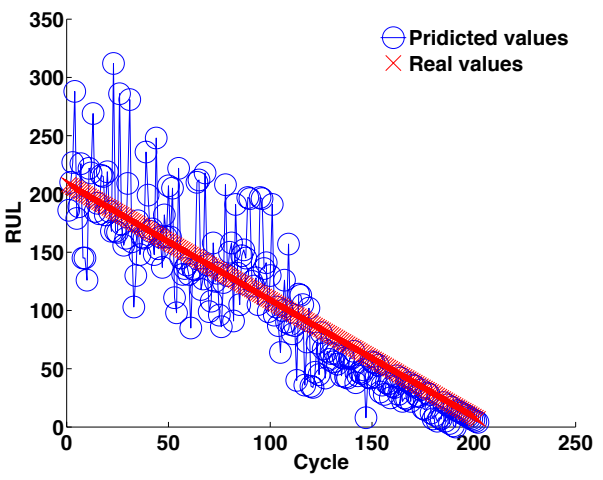

(a) RUL of engine 34 .

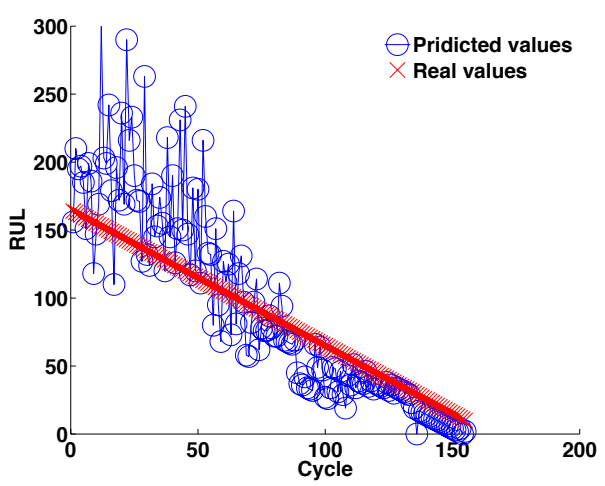

(c) RUL of engine 42 .

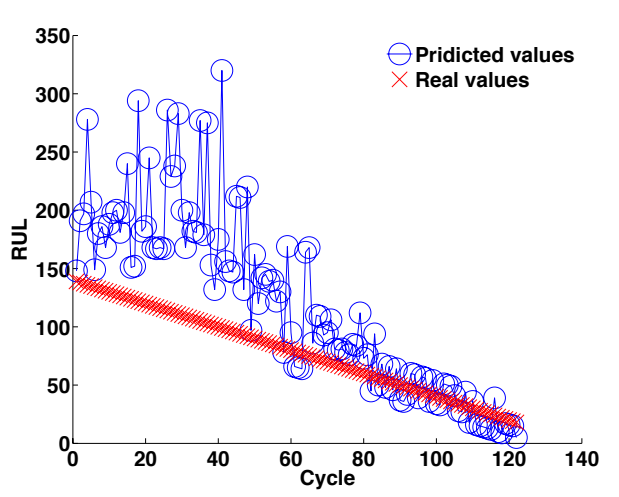

(b) RUL of engine 41.

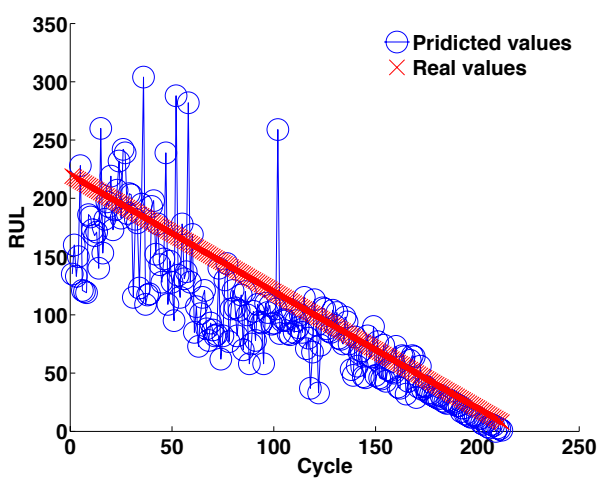

(d) RUL of engine 81 .

Fig. 9: Results of predicting the RUL at all cycles for 4 engines.

is then divided into training and testing data set as depicted in Table 1 and Table 2, respectively. Only 31 battery data sets are used in this experiment as three batteries, namely B0018, B0041 and B0053, do not have any similar data sets with the same end of life.

Variable selection: One of the results of the selection algorithm is the pair of variables $\{6,11\}$, i.e. the voltage measured at discharge and the capacity of the battery (Figure 10). The selected pair is interesting because the two variables are correlated. Indeed, the capacity is related to the battery health as the decrease in the capacity indicates health degradation.

Health indicator: Four features are extracted from each trend at each time and labeled with end of life time to be saved in the offline database. Figure 5 shows two of the four health indicators for the battery B0005. The indicators are monotonic and show how the relation between the end of life and the extracted trend changes through the time. 
Table 1: Training data sets with three folds.

\begin{tabular}{llll}
\hline Fold \#1 & Fold \#2 & Fold \#3 & EOL \\
\hline B0006 & B0005 & B0005 & 168 \\
B0007 & B0007 & B0006 & 168 \\
B0026 & B0025 & B0025 & 28 \\
B0027 & B0026 & B0026 & 28 \\
B0028 & B0027 & B0028 & 28 \\
B0030 & B0029 & B0029 & 40 \\
B0031 & B0031 & B0030 & 40 \\
B0032 & B0032 & B0031 & 40 \\
B0034 & B0033 & B0033 & 197 \\
B0036 & B0036 & B0034 & 197 \\
B0039 & B0038 & B0038 & 47 \\
B0040 & B0040 & B0039 & 47 \\
B0043 & B0042 & B0042 & 112 \\
B0044 & B0044 & B0043 & 112 \\
B0045 & B0045 & B0045 & 72 \\
B0047 & B0046 & B0046 & 72 \\
B0048 & B0048 & B0047 & 72 \\
B0050 & B0049 & B0049 & 25 \\
B0051 & B0050 & B0050 & 25 \\
B0052 & B0051 & B0052 & 25 \\
B0055 & B0054 & B0054 & 102 \\
B0056 & B0056 & B0055 & 102 \\
\hline
\end{tabular}

Table 2: Testing data sets with three folds.

\begin{tabular}{llll}
\hline Fold \#1 & Fold \#2 & Fold \#3 & EOL \\
\hline B0005 & B0006 & B0007 & 168 \\
B0025 & B0028 & B0027 & 28 \\
B0029 & B0030 & B0032 & 40 \\
B0033 & B0034 & B0036 & 197 \\
B0038 & B0039 & B0040 & 47 \\
B0042 & B0043 & B0044 & 112 \\
B0046 & B0047 & B0048 & 72 \\
B0049 & B0052 & B0051 & 25 \\
B0054 & B0055 & B0056 & 102 \\
\hline
\end{tabular}

Prediction results: To assess the performance of the proposed method, MAPE is calculated for all cycles of each battery (Figure 11). The average MAPE per fold is calculated as follows:

$$
M A P E_{f}=\frac{1}{n} \times \sum_{i=1}^{n} M A P E_{i, f}
$$

where $M A P E_{f}$ is the average MAPE for a complete fold, $M A P E_{i, f}$ is the MAPE for test battery $i$ in fold $f$. The final results are calculated and summarized in Table 3.

Figure 11a shows plot of the predicted RUL for all cycles for battery B0005. It can be seen that the prediction accuracy increases with time, i.e, the longer the test trend is the higher the predication accuracy. Figure 11b shows a plot 


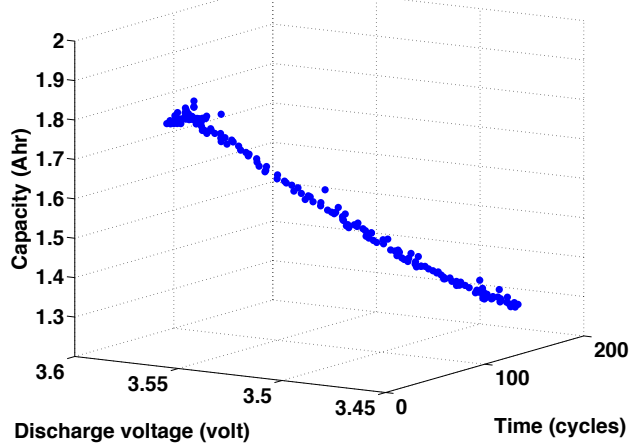

Fig. 10: Selected pair of variables from the NASA battery B0005.

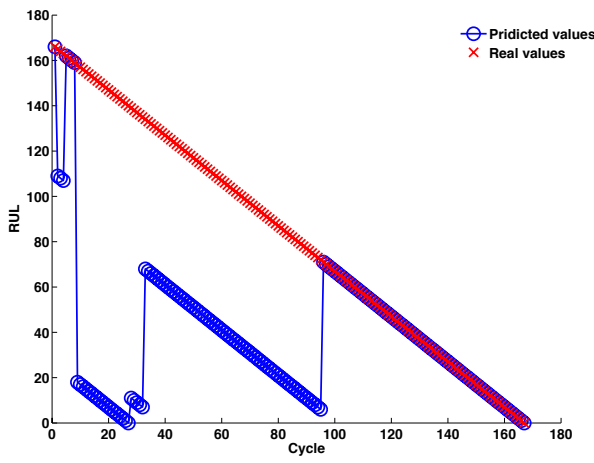

(a) RUL of battery B0005.

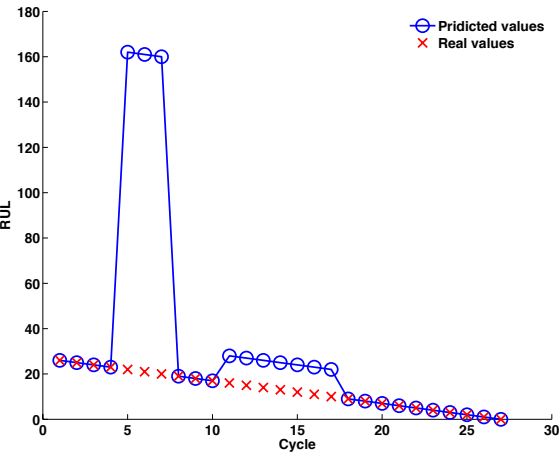

(b) RUL of battery B0025.

Fig. 11: Results of predicting the RUL at all cycles for 2 batteries.

Table 3: Mean absolute percentage error for the NASA battery data sets.

\begin{tabular}{llll}
\hline Fold \#1 & Fold \#2 & Fold \#3 & Average \\
\hline $28.0493 \%$ & $26.3089 \%$ & $28.3536 \%$ & $27.5706 \%$ \\
\hline
\end{tabular}

of the RUL predicted for the battery B0025. Only 10 cycles were considered as late prediction. However, the error was decreasing at the later cycles.

\section{Conclusion}

In this paper a data driven method for RUL prediction based on a Bayesian approach is proposed. The method builds on unsupervised selection of interesting variables from the input offline signals. It constructs representative features that can be used as health indicators. The method represents the current sta- 
tus of the online signals as well as the uncertainty about the predictions in a probabilistic form.

The performance of the proposed method is evaluated using two data sets, namely, turbofan engines and lithium-ion battery data downloaded form the NASA prognostic center of excellence website. The prediction results show low MAPE error for both applications.

For future work, the proposed method should consider the data sets with no training samples in the database, such as the case with battery data sets. Also, it should be tested using data sets with variable operating conditions and after introducing maintenance interventions. Different classification/regression models should be tested in the proposed framework.

\section{References}

1. Neil Montgomery, Dragan Banjevic, Andrew K. S. Jardine. "Minor maintenance actions and their impact on diagnostic and prognostic CBM models". Journal of Intelligent Manufacturing, volume 23, issue 2, pages 303-311, April 2012. DOI: 10.1007/s10845-009-0352-0

2. Ranganath Kothamasu, Samuel H. Huang, William H. VerDuin. "System health monitoring and prognostics a review of current paradigms and practices." The International Journal of Advanced Manufacturing Technology, volume 28, issue 9-10, pages 1012-1024, July 2006. DOI: 10.1007/s00170-004-2131-6.

3. Sarah S. Soh, Nor. H. M. Radzi, Habibollah Haron. "Review on Scheduling Techniques of Preventive Maintenance Activities of Railway." Fourth International Conference on Computational Intelligence, Modelling and Simulation (CIMSiM), pages 310-315, 25-27 Sept. 2012, Kuantan, Malaysia. DOI: 10.1109/CIMSim.2012.56.

4. Ying Peng, Ming Dong, Ming Jian Zuo. "Current status of machine prognostics in condition-based maintenance: a review." The International Journal of Advanced Manufacturing Technology, volume 50, issue 1-4, pages 297-313, September 2010. DOI: 10.1007/s00170-009-2482-0.

5. Andrew K.S. Jardine, Daming Lin, Dragan Banjevic. "A review on machinery diagnostics and prognostics implementing condition-based maintenance." Mechanical Systems and Signal Processing, volume 20, issue 7, pages 14831510, October 2006. DOI: dx.doi.org/10.1016/j.ymssp.2005.09.012.

6. Kamal Medjaher, Diego A. Tobon-Mejia, Noureddine Zerhouni. "Remaining Useful Life Estimation of Critical Components With Application to Bearings." IEEE Transactions on Reliability, volume 61, number 2, pages 292-302, June 2012. DOI: 10.1109/TR.2012.2194175.

7. Jianfei Dong, Michel Verhaegen, Fredrik Gustafsson. "Robust Fault Detection With Statistical Uncertainty in Identified Parameters," IEEE Transactions on Signal Processing, volume 60, number 10, pages 5064-5076, October. 2012. DOI: 10.1109/TSP.2012.2208638.

8. Kihoon Choi, Satnam Singh, Anuradha Kodali, Krishna R. Pattipati, John W. Sheppard, Setu Madhavi Namburu, Shunsuke Chigusa, Danil V. Prokhorov, Liu Qiao. "Novel Classifier Fusion Approaches for Fault Diagnosis in Automotive Systems," IEEE Transactions on Instrumentation and Measurement, volume 58, number 3, pages 602-611, March 2009. DOI: 10.1109/TIM.2008.2004340.

9. Diego A. Tobon-Mejia, Kamal Medjaher, Noureddine Zerhouni, Gerard Tripot. "A DataDriven Failure Prognostic Method based on Mixture of Gaussians Hidden Markov Models." IEEE Transactions on Reliability, volume 61, number 2, pages 491-503, June 2012. DOI: $10.1109 /$ TR.2012.2194177.

10. Iyer, N., Goebel, K., Bonissone, P., "Framework for post-prognostic decision support," IEEE Aerospace Conference, volume 9, number 1, pages 3962-3971, March 2006. DOI: 10.1109/AERO.2006.1656108 
11. Kamran Javed, Rafael Gouriveau, Noureddine Zerhouni, Patrick Nectoux. "A feature extraction procedure based on trigonometric functions and cumulative descriptors to enhance prognostics modeling." IEEE Prognostics and Health Management (PHM) Conference, volume 1, number 7, pages 24-27, June 2013. DOI: 10.1109/ICPHM.2013.6621413.

12. Bhaskar Saha, Kai Goebel. "Uncertainty Management for Diagnostics and Prognostics of Batteries using Bayesian Techniques," IEEE Aerospace Conference, volume 1, number 8, pages 1-8, March 2008. DOI: 10.1109/AERO.2008.4526631.

13. Pal, S., Heyns, P. S., Freyer, B. H., Theron, N. J., \& Pal, S. K. (2011). Tool wear monitoring and selection of optimum cutting conditions with progressive tool wear effect and input uncertainties. Journal of Intelligent Manufacturing, 22, 491504.

14. Aiwina Heng, Sheng Zhang, Andy C.C. Tan, Joseph Mathew. "Rotating machinery prognostics: State of the art, challenges and opportunities." Mechanical Systems and Signal Processing, volume 23, issue 3, pages 724-739, April 2009. DOI: dx.doi.org/10.1016/j.ymssp.2008.06.009.

15. R. Isermann, Fault-Diagnosis Systems: An Introduction from Fault Detection to Fault Tolerance. Heidelberg: Springer-Verlag, Heidelberg, 2006.

16. G. Vachtsevanos, F. Lewis, M. Roemer, A. Hess, B. Wu, Intelligent Fault Diagnosis and Prognosis for Engineering Systems, John Wiley and Sons Inc., Hoboken, New Jersey, 2006

17. J. Luo, M. Namburu, K. Pattipati, L. Qiao, M. Kawamoto, S. Chigusa, Model-Based Prognostic Techniques, Anaheim, CA, United States: 2003, Institute of Electrical and Electronics Engineers Inc., Piscataway, NJ, United States, 2003, pages 330-340.

18. Fakher Chaari, Tahar Fakhfakh, Mohamed Haddar. "Analytical modelling of spur gear tooth crack and influence on gearmesh stiffness." European Journal of Mechanics - A/Solids, volume 28, issue 3, pages 461-468, June 2009. DOI: dx.doi.org/10.1016/j.euromechsol.2008.07.007.

19. Zhenyou Zhang, Yi Wang, Kesheng Wang. "Fault diagnosis and prognosis using wavelet packet decomposition, Fourier transform and artificial neural network". Journal of Intelligent Manufacturing, volume 24, issue 6, pages 1213-1227, December 2013. DOI: $10.1007 / \mathrm{s} 10845-012-0657-2$

20. He, D., Li, R., Bechhoefer, E. (2012). Stochastic modeling of damage physics for mechanical component prognostics using condition indicators. Journal of Intelligent Manufacturing, 23, 221226.

21. M.A. Schwabacher, A Survey of Data-Driven Prognostic, Infotech@Aerospace, pages 26 - 29 September 2005, Arlington, Virginia.

22. G.E.P. Box, G.M. Jenkins, Time Series Analysis: Forecasting and Control, Holden-Day, San Francisco, 1976

23. F. Lewis, Applied Optimal Control and Estimation: Digital Design and Implementation, Prentice-Hall, Englewood Cliffs, NJ, 1992.

24. R.S. Tsay, Time series and forecasting: brief history and future research, Journal of the American Statistical Association 95 (450) (2000), pages 638-643.

25. W.Wu, J.Hu, J. Zhang, Prognostics of machine health condition using an improved ARIMA-based prediction method, IEEE, Harbin, China, 2007, pages 1062-1067.

26. J. Yan, M. Koc, J. Lee, A prognostic algorithm for machine performance assessment and its application, Production Planning and Control 76 (2004), pages 796-801.

27. J. Lee, J. Ni, D. Djurdjanovic, H. Qiu, H. Liao, Intelligent prognostics tools and emaintenance, Computers in Industry 57 (6) (2006), pages 476-489.

28. N. Gebraeel, M. Lawley, R. Liu, V. Parmeshwaran, Residual life predictions from vibration-based degradation signals: a neural network approach, IEEE Transactions on Industrial Electronics 51 (3) (2004), pages 694-700.

29. B. Satish, N.D.R. Sarma, A fuzzy BP approach for diagnosis and prognosis of bearing faults in induction motors, IEEE Power Engineering Society General Meeting, IEEE, 2005, pages 2291-2294.

30. R. Huang, L. Xi, X. Li, C. Richard Liu, H. Qiu, J. Lee, Residual life predictions for ball bearings based on self-organizing map and back propagation neural network methods, Mechanical Systems and Signal Processing 21 (1) (2007), pages 193-207.

31. Z. Lei, L. Xingshan, Y. Jinsong, G. ZhanBao, A genetic training algorithm of wavelet neural networks for fault prognostics in condition based maintenance, in: Proceedings of 
the Eighth International Conference on Electronic Measurement and Instruments, IEEE, 2007, pages 584-589.

32. A.P. Vassilopoulos, E.F. Georgopoulos, V. Dionysopoulos, Artificial neural networks in spectrum fatigue life prediction of composite materials, International Journal of Fatigue 29 (1) (2007), pages 20-29.

33. Zhigang Tian. "An artificial neural network method for remaining useful life prediction of equipment subject to condition monitoring". Journal of Intelligent Manufacturing, volume 23, issue 2, pages 227-237, April 2012. DOI: 10.1007/s10845-009-0356-9

34. Kamran Javed, Rafael Gouriveau, Noureddine Zerhouni. "Novel failure prognostics approach with dynamic thresholds for machine degradation." 39th Annual Conference of the IEEE Industrial Electronics Society, (IECON), pages 4404-4409, 10-13 November 2013 DOI: $10.1109 /$ IECON.2013.6699844

35. Brezak, D., Majetic, D., Udiljak, T., Kasac, J. (2012). Tool wear estimation using an analytic fuzzy classifier and support vector machines. Journal of Intelligent Manufacturing, 23, 797809 .

36. Gajate, A., Haber, R., Del Toro, R., Vega, P., Bustillo, A. (2012). Tool wear monitoring using neuro-fuzzy techniques: A comparative study in a turning process. Journal of Intelligent Manufacturing, 23, 869882.

37. Purushothaman, S. (2010). Tool wear monitoring using artificial neural network based on extended Kalman filter weight updation with transformed input patterns. Journal of Intelligent Manufacturing, 21, 717730.

38. Yeo, S. H., Khoo, L. P., Neo, S. S. (2000). Tool condition monitoring using reflectance of chip surface and neural network. Journal of Intelligent Manufacturing, 11, 507514.

39. G.P. Zhang. "Time series forecasting using a hybrid ARIMA and neural network model", Neurocomputing 50 (2003), pages 159-175.

40. N. Gorjian, L. Ma, M. Mittinty, P. Yarlagadda, and Y. Sun. Review on Degradation Models in Reliability Analysis, Proceedings of the 4th World Congress on Engineering Asset Management, 2009, 28-30 Sept, Athens, Greece.

41. T. Benkedjouh, K. Medjaher, N. Zerhouni, S. Rechak. "Health assessment and life prediction of cutting tools based on support vector regression". Journal of Intelligent Manufacturing, article published online 19 April 2013, DOI: 10.1007/s10845-013-0774-6

42. Tianyi Wang; Jianbo Yu; Siegel, D.; Lee, J. "A similarity-based prognostics approach for Remaining Useful Life estimation of engineered systems." IEEE International Conference on Prognostics and Health Management, volume 1, number 6, pages 6-9, October 2008. DOI: 10.1109/PHM.2008.4711421

43. Emmanuel Ramasso, Michle Rombaut, and Noureddine Zerhouni. "Joint Prediction of Continuous and Discrete States in Time-Series Based on Belief Functions." IEEE Transactions on Cybernetics, voume 43, number 1, pages 37-50, Feb. 2013. DOI: 10.1109/TSMCB.2012.2198882.

44. J.Z. Sikorska, M. Hodkiewicz, L. Ma. "Prognostic modelling options for remaining useful life estimation by industry." Mechanical Systems and Signal Processing, volume 25, issue 5, pages 1803-1836, July 2011. DOI: dx.doi.org/10.1016/j.ymssp.2005.09.012.

45. Mosallam A., Medjaher K., Zerhouni, N. "Nonparametric time series modelling for industrial prognostics and health management." The International Journal of Advanced Manufacturing Technology, volume 69, issue 5, pages 1685-1699, November 2013. DOI: 10.1007/s00170-013-5065-z.

46. Mosallam, A., Byttner, S., Svensson, M., T., R.: "Nonlinear relation mining for maintenance prediction". IEEE Aerospace Conference, pages 1-9, March 2011. DOI: 10.1109/AERO.2011.5747581

47. Huang, N.E., Shen, Z., Long, S.R., Wu, M.C., Shih, H.H., Zheng, Q., Yen, N.C., Tung, C.C., Liu, H.H. "The empirical mode decomposition and the hilbert spectrum for nonlinear and non-stationary time series analysis." Proceedings of the Royal Society of London Series A. Mathematical, Physical and Engineering Sciences, pages 903-995, 1998.

48. Nectoux, P., Gouriveau, R., Medjaher, K., Ramasso, E., Chebel-Morello, B., Zerhouni, N., Varnier, C. "Pronostia: An experimental platform for bearings accelerated degradation tests." IEEE International Conference on Prognostics and Health Management, 2012, Denver, Colorado, USA. 
49. Marco Trincavelli, Silvia Coradeschi, Amy Loutfi, Odour classification system for continuous monitoring applications, Sensors and Actuators B: Chemical, Volume 139, Issue 2, 4 June 2009, Pages 265-273, ISSN 0925-4005, doi: 10.1016/j.snb.2009.03.018.

50. Tangbin Xia, Lifeng Xi, Xiaojun Zhou, Jay Lee. "Dynamic maintenance decision-making for series-parallel hybrid multi-unit manufacturing system based on MAM-MTW methodology". European Journal of Operational Research. (2012) 221, 231-240.

51. Lin Li, Jun Ni. "Short-term decision support system for maintenance task prioritization". International Journal of Production Economics. (2009) 121 (1), 195-202.

52. B. Saha and K. Goebel (2007). "Battery Data Set", NASA Ames Prognostics Data Repositoryhttp://ti.arc.nasa.gov/project/prognostic-data-repository], NASA Ames, Moffett Field, CA.

53. A. Saxena and K. Goebel (2008). "C-MAPSS Data Set", NASA Ames Prognostics Data Repository, [http://ti.arc.nasa.gov/project/prognostic-data-repository], NASA Ames, Moffett Field, CA 